\title{
An Upper-Level Ontological Model for Engineering Design Performance Domain
}

\author{
Vadim Ermolayev $^{1}$, Natalya Keberle ${ }^{1}$, Wolf-Ekkehard Matzke ${ }^{2}$ \\ ${ }^{1}$ Department of IT, Zaporozhye National University, Zhukovskogo 66, 69063, \\ Zaporozhye, Ukraine \\ vadim@ermolayev.com,kenga@zsu.zp.ua \\ ${ }^{2}$ Cadence Design Systems, GmbH, Mozartstr. 2 D-85622 Feldkirchen, Germany \\ wolf@cadence.com
}

\begin{abstract}
The paper presents PSI ${ }^{1}$ Meta-Ontology - an upper level lightweight descriptive model for the set of the Core ontologies of PSI Suite. While PSI Suite is an interlinked modular library of ontologies describing the domain of engineering design performance in microelectronics, PSI Meta-Ontology is more domain-independent. It is an upper-level model of stateful creative dynamic processes, pro-active agents, and objects situated in nested dynamic environments based on formal representation of time, events, and happenings. It may be used as an upper-level theory for domain ontologies in different application domains having common features. PSI Meta-Ontology is designed as a semantic bridge facilitating to mapping PSI Domain ontologies to abstract ontological foundations and common sense. It is also used as semantic "glue" for bridging PSI ontologies with other theories, widely accepted in the domains where processes, states, and participating objects are the major entities. These mappings and semantic bridges are supposed to ease the commitment of potential users to PSI Suite. PSI Meta-Ontology is also used as a "proxy" for different kinds of evaluation of PSI ontologies in frame of our "shaker modeling" methodology for ontology refinement.
\end{abstract}

\section{Introduction}

PSI project deals with the development of the methodology and the toolset for assessing, predicting, and optimizing the performance of engineering design systems in microelectronics. Though design technology in microelectronics domain is well defined, many factors make design processes highly stochastic, non-deterministic, structurally ramified, time-bound - in a phrase, loosely defined and highly dynamic. The examples of such factors are: human factor, innovative character, the pace of technology change, the peculiarities of the market and customer requirements, etc. PSI uses simulation to observe and predict the course of a Dynamic Engineering

${ }^{1}$ Performance Simulation Initiative (PSI) is a research and development project of Cadence Design Systems, GmbH. 
Design Process (DEDP) in dynamics with sufficient detail for making assessments grounded. Simulation allows playing "what-if" games to model the unpredictable character of the real business world of microelectronic design.

Due to the omnipresence of the mentioned factors which complicate the development of a DEDP in time, the social behavior of the project team, and the influence of the environment, fine grained and complete knowledge of a process is the key intellectual asset which allows PSI methodology be convincing. This knowledge is formalized using the Suite of PSI ontologies. If someone imagines an arbitrary process of designing something, most certainly he or she will think in terms of: a goal - the state of affairs to be reached; an action which may bring the process closer to its goal; an object to apply actions to; a designer who acts and applies actions to objects; an instrument to be used by an actor to execute actions; and an environment in which the process occurs. All these interact in dynamics - depending on time and on events which manifest the changes in a design system which is the environment of a DEDP. The structure of the Suite of PSI Ontologies reflects this approach. It comprises six cross-linked Core ontologies: Time ontology; Environment, Event and Happening ontology; Actor ontology; Project ontology; Process and Process Pattern ontologies; and Design Artifact ontology. The "corolla" of this Core is formed by Extension ontologies collaboratively developed in PSI and PRODUKTIV $+^{2}$ projects. The most important Extensions are: Resource ontology with Tool package, Generic Negotiation ontology.

PSI Meta-Ontology is the upper-level part of PSI Suite of Ontologies. Its main function is putting the components of the Suite in line with the commonly accepted metaphysical and cognitive framework of the common sense represented by chosen reference ontologies. One more objective of introducing the upper-level of the Suite is providing semantic bridge to mainstream enterprise, business, and process modeling frameworks. Bridging PSI ontologies to these mainstream theories of process knowledge representation facilitates to easier commitment of engineering design domain professionals to the Suite. Meta-Ontology also plays an integration and harmonization role in PSI Suite because it represents a rather domain-independent upper-level descriptive theory based on formal principles for harmonizing and integrating the underlying domain dependent modules with other relevant ontologies. In addition to being the semantic "glue" between the Suite and the outer world of knowledge representation the Meta-Ontology plays an important role in the methodology of knowledge engineering in PSI. It is the resource which is intensively used in the refinement and the evaluation of PSI Core Ontologies.

The rest of the paper is structured as follows. Section 2 outlines requirements and objectives which shaped out our upper-level model. Section 3 puts PSI upper-level ontology in the context of related work and presents our reasons for ontological choices. Section 4 presents PSI Meta-Ontology by outlining its taxonomy and discussing the semantic context of its key concepts in detail. Section 5 sketches our ontology engineering methodology. Section 6 reports on the implementation and

2 PRODUKTIV+ (Referenzsystem zur Messung der Produktivität beim Entwurf nanoelektronischer Systeme) is the R\&D project funded by the German Bundesministerium für Bildung und Forschung (BMBF). 
evaluation of PSI Meta-Ontology. Finally, concluding remarks and our plans for future work are given in Section 7.

\section{Modeling Requirements}

PSI project aims at developing models, methodologies, and software tools providing for rigorous engineering treatment of performance and performance management. PSI performance modeling and management approach focuses on performance as a proactive action. A fine-grained dynamic model of a DEDP and a design system is therefore developed. PSI approach considers that performance is embodied in its environment and is controlled by the associated performance management process [1].

A DEDP is a goal-directed process of transforming the representations of a design artifact in stateful nested environments. An environment comprises design artifact representations, resources, tools, and actors who perform actions to transform design artifacts using tools, consume resources. Actions are admissible in particular environment states and may be atomic or compound, state-transitive or iterative, dependent or independent on other actions. The components of an environment may generate internal events or may be influenced by external events. Events may have causal dependencies. A DEDP is a problem solving process which goals, partial goals, and environments may change dynamically. In PSI a decision taking procedure is associated with each state to allow environments adjust the course of a DEDP taking these changes into account. Decisions are taken by actors modeled by software agents.

PSI software tools are developed [2] for assisting project managers to make robust planning, monitoring, and management of their design projects aiming at reaching best possible performance. Grounded decisions in planning are based on the knowledge base of project logs accomplished in the past. These logs provide vast and finely grained records of the performance of the accomplished projects and may be used for simulating the behavior of the design system in response to different influences. At project execution phase PSI software may be used for predicting the behavior of the design system in the future based on the record of the partially accomplished DEDP, the knowledge about its environment(s), and performance simulations.

Mentioned functionalities may only be implemented if a rich and expressive domain model is used. This model should be capable of facilitating agents reasoning about environments, events, and actions employed in decision taking procedures enacted at environmental states. These sorts of commonsense reasoning require ontological representations of time [3], environments, events and their subjective perceptions [4], processes, actions, actors, design artifacts, resources, tools [5-7]. The models of these domain aspects form the Core and the Extensions of PSI Suite of Ontologies v.2.1 [6] and v.2.2 [7]. 


\section{Related Work and Modeling Choices}

PSI Meta-Ontology is the upper-level part of PSI Suite of Ontologies [6, 7]. Its main function is putting the components of the Suite in line with the commonly accepted metaphysical and cognitive framework of the common sense represented by several reference ontologies like Suggested Upper Merged Ontology (SUMO) [8], Descriptive Ontology for Linguistic and Cognitive Engineering (DOLCE) [9] and highly reputable linguistic resources like WordNet Linguistic Ontology (WordNet) [10]. One more objective of introducing the upper-level of the Suite is providing semantic bridges to mainstream enterprise, business, and process modeling frameworks like the Enterprise Ontology (EO) [11], Toronto Virtual Enterprise Ontology (TOVE) [12], Process Specification Language (PSL) [13].

In difference to the mentioned enterprise, business, and process modeling frameworks, which are, to a certain extent, domain independent (TOVE, PSL) or model manufacturing Domain (EO), PSI Meta-Ontology defines an upper-level theory for the domain of engineering design processes and environments. As many foundational ontologies PSI Meta-Ontology has a clear cognitive orientation in the sense that it does not pretend being strictly and rigorously referential to the theories describing nature. Instead, it captures ontological categories and contexts based on human common sense reflecting socially dominant views on the Domain characteristic at least to engineering design professionals. As such, the categories introduced in PSI Meta-Ontology are not related to the intrinsic nature of the world but are rather thought of as "cognitive artifacts ultimately depending on human perception, cultural imprints and social conventions" [9]. Therefore, these categories assist in making already formed conceptualizations of PSI Suite of Ontologies explicit and referenced by the common sense. PSI Meta-Ontology also plays an integration and harmonization role of a foundational ontology [14] because it represents a rather domain-independent descriptive theory based on formal principles for harmonizing and integrating the underlying domain dependent modules with other relevant ontologies.

In contrast to foundational ontologies PSI Meta-Ontology is not foundational in the sense that it is not a profound and a complete theory in philosophical or, more precisely, cognitivistic sense. For example, PSI Meta-Ontology does not deal with many problems characteristic for foundational theories like: differences between abstract and concrete objects, particulars and universals; spatio-temporal colocalization of things; mereological axiomatization; etc. It also does not provide rich axiomatic sets for rigorously describing the semantics of the contained entities. Instead, other highly reputable foundational ontologies are used as reference sources for defining PSI Meta-Ontology components. The mappings of these components to those reference sources are explicitly specified. Choosing the most appropriate reference foundational ontologies among possible candidates is not an easy task because it requires ontological commitment to the chosen ontologies and their ontological choices. Typical ontological choices (also called meta-criteria) are: (i) Descriptivism vs. Revisionarism; (ii) Multiplicativism vs. Reductionism; (ii) Possibilism vs. Actualism; (iv) Endurantism vs. Perdurantism. A good comparative analysis of several well known foundational ontologies and their ontological choices has been undertaken in SmartWeb project [15]. Five most promising candidates 
among approximately a dozen available worldwide has been analyzed: Basic Formal Ontology (BFO), DOLCE, Object-Centered High-level Reference Ontology (OCHRE), OpenCYC, and SUMO. The results are given in Table 1. Typical ontological choices in line with modeling requirements of PSI project are discussed below. Based on this discussion our choice of reference foundational ontologies for the design of PSI Meta-Ontology is made.

Table 1. Foundational ontologies and their ontological choices [15].

\begin{tabular}{|l|c|c|c|c|c|}
\hline \multicolumn{1}{|c|}{ Ontology } & BFO & DOLCE & OCHRE & OpenCYC & SUMO \\
\hline Alternative & - & + & - & + & + \\
\hline Descriptivism & - & + & unclear & unclear & + \\
\hline Multiplicativism & + & - & - & unclear & unclear \\
\hline Actualism & + & + & - & unclear & + \\
\hline Perdurantism & &
\end{tabular}

Legend: + - the ontology supports the ontological choice; - - the ontology does not support the ontological choice; unclear - it is not clear if the ontology supports the ontological choice.

Descriptivism vs. Revisionarism A descriptive ontology aims at describing the ontological assumptions based on the surface structure of natural language and human common sense. For example, a descriptive ontology distinguishes between physical and abstract objects based on the human common sense perception of these categories. It is common to consider that a physical object is a category of things which have tangible physical properties, can be sensed, are extended in space and time. On the contrary, an abstract object does not possess the abovementioned properties. A revisionary ontology is committed to capture the intrinsic nature of the world. As a consequence, such a commitment may impose that only entities extended in space and time exist. Though we refrain from modeling abstract things in PSI as much as possible ${ }^{3}$, we still have to model immaterial things which are not made of matter, do not possess spatial properties, etc. Therefore, revisionarism would have been a wrong choice for PSI. PSI Meta-Ontology is a descriptive ontology and has to be based on a descriptive foundational ontology like DOLCE, OpenCYC, or SUMO.

Multiplicativism vs. Reductionism A multiplicative ontology allows different entities to be co-localized in the same space-time. The difference of these entities means that they have different essential properties. For example, a silicon wafer of a chip (a material object) and a definite amount of silicon this wafer is made of (an amount of matter) are co-localized in space-time for the whole life of this particular chip. Reductionistic ontology postulates that each space-time location contains at most one object. Differences in essential properties are regarded as being linked to different points of view from which one can look at the same spatio-temporal entity. Reductionistic approach therefore extracts all essential properties different from spatio-temporal ones from entities and places them to the views on these entities. In PSI it is considered that an entity possesses all its essential properties and the views on an entity may reveal different subsets of these properties depending on the point of view. For example, an agent may be (i) a model of one physical person - a designer; (ii) a model of a group of designers working on one design project - a development

\footnotetext{
${ }^{3}$ All concepts of PSI Meta-Ontology are not abstract - Fig. 1.
} 
team. PSI Meta-Ontology should therefore be a multiplicative ontology - like DOLCE or SUMO.

Possibilism vs. Actualism An actualistic ontology postulates that everything that exists is actual. Things that are not actual and, therefore, do not exist may be withdrawn from consideration. Different forms of possibilism are based on different ways of the denial of this postulate. For example our beliefs, which are hypotheses based on incomplete, partial knowledge about the world, are very often roughly equally believed possible alternatives. Considering such alternatives is characteristic to human common sense and cognition. Committing to possibilism means being able to represent possibilia - possible alternative entities in a domain corresponding to different modalities in different possible worlds. Possibilism is particularly useful in reasoning about future courses of processes and about actions [16]. PSI MetaOntology has to be capable of modeling possibilia. For example, a design process depending on the future events in its environment may take one of the possible alternative courses. These alternative courses should all be considered and analyzed for choosing the best possible one to follow. Hence, we have to commit to possibilism of a foundational ontology like DOLCE or OCHRE.

Endurantism vs. Perdurantism A fundamental ontological choice is the commitment to a way of modeling changes of things in time. Endurantism (also called $3 \mathrm{D}$ paradigm) postulates that all things do not change in time in the sense that all the proper parts of an entity (a whole) are present in this whole at any moment of the existence of this whole. Differently to that, perdurantism (also called 4D paradigm) assumes that entities may have different parts at different moments of their existence - meaning that entities have both spatial and temporal constituents. PSI MetaOntology needs to model both endurants and perdurants. Indeed, many of the concepts characteristic to engineering design always contain all their parts, but many other of them are composed of temporally different parts - like phases in a design process. Therefore, a reference foundational ontology for PSI Meta-Ontology should be based on $4 \mathrm{D}$ paradigm, comprising $3 \mathrm{D}$ as a particular case. Such ontologies are BFO, DOLCE, and SUMO.

The requirements analyzed above reveal that only DOLCE commits to all the ontological choices required by PSI. SUMO provides all the necessary features except possibilism. This is why we fully commit to the foundational framework of the upper taxonomical level of DOLCE in our design of PSI Meta-Ontology. We also use SUMO extended by WordNet as a target for mapping the concepts of PSI MetaOntology and PSI Core Ontologies because SUMO+WordNet is probably the most prominent linguistic resource describing the semantics of human common sense.

\section{PSI Upper-Level Ontology}

The postulates, assumptions, objectives, and ontological choices of PSI modeling approach were presented in Sections 2 and 3. Here the semantic contexts [17] of several key concepts of PSI Meta-Ontology are discussed in detail: a Process, a State, an Object, an Agent, and a Rule. Complete specification of the ontology may be found in [18]. Fig. 1 pictures the taxonomy of PSI Meta-Ontology. 


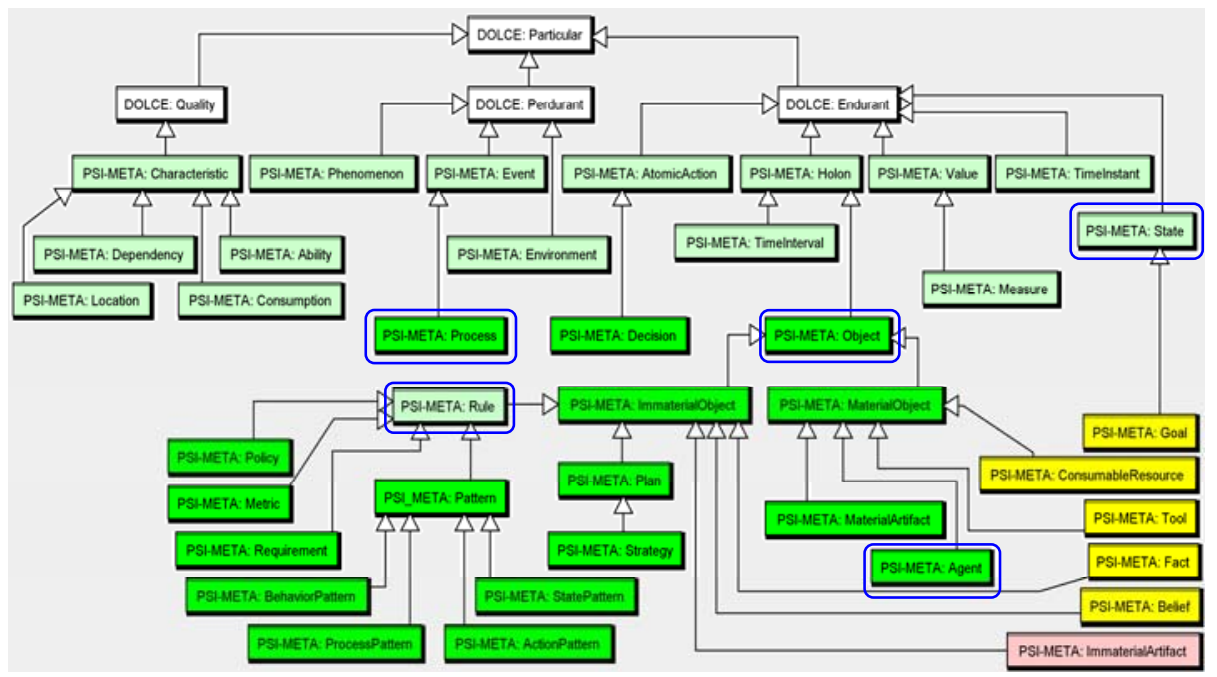

Legend:

(i) Concepts are colored reflecting classification by OntoClean property types (Section 6): $\square$ - category, $\square$ - type, $\square$ - quasi-type, $\square$ - material role, $\square$ - mixin.

Categories, types, and quasi-types form the backbone taxonomy

(ii) Semantic contexts of the concepts in rounded rectangles ( $\square$ ) are discussed in detail

Fig. 1. The taxonomy of PSI Meta-Ontology.

A Process (Fig. 2a) is a specialization of an Event ${ }^{4}$ which is stateful and possesses pro-active character. A Process has its Environment - the part of the world which may influence the course of the Process or may be changed in the course of the Process. A Process is pro-actively directed by the Agent who manages it. Pro-activeness of the Agent is understood in the sense that the Agent pursues a particular Goal in the managed Process. This Goal is the State of the Environment which the Agent desires to make reached. It should also be mentioned that the change in the Environment is not produced by the Process, but by the entities who act in this process - those Agents who execute AtomicActions wrapped by the Process. In general, it is considered that changes may only be applied by Agents through execution of AtomicActions. For example, it is wrong to say that a multimedia controller layout has been designed by the process of logical design. In fact the appearance of the layout for the multimedia controller in a certain state of the Environment (the measurable change in the Environment) has been achieved by the team of Agents who executed a particular sequence of AtomicActions. By that the Agents applied the sequence of particular changes to the Environment and guided the environment through the sequence of States towards the Goal. Processes in an engineering Environment can not connect any arbitrary State to any other arbitrary State because it is senseless with respect to the technology or the methodology. Some sequences of States may therefore be withdrawn from the engineering design routine and some other sequences of States

4 Detailed discussion of PSI approach to modeling Environments, Events, and event Happenings subjectively perceived by Agents is presented in our parallel paper [4]. 


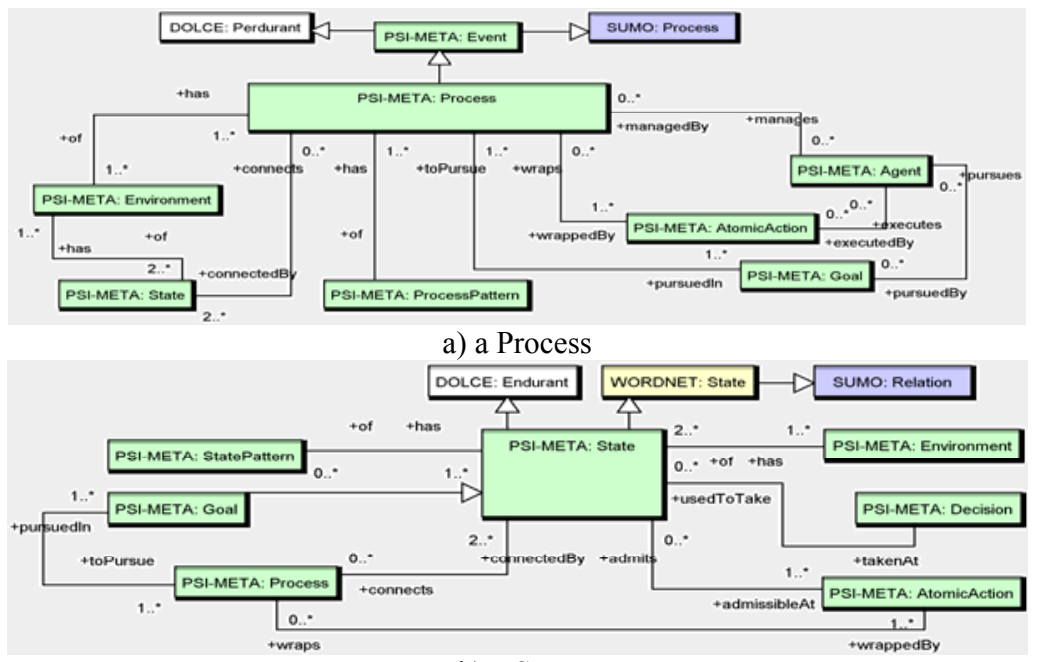

b) a State

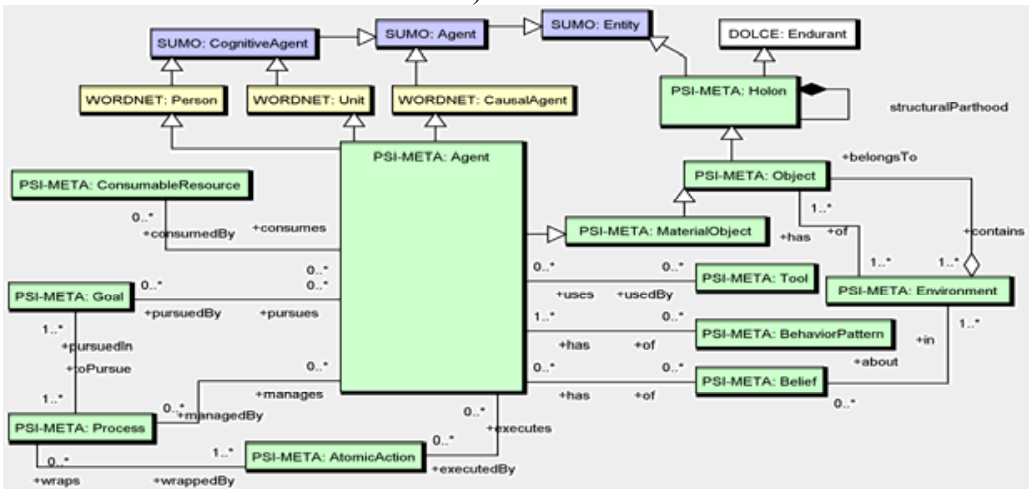

c) an Object and an Agent

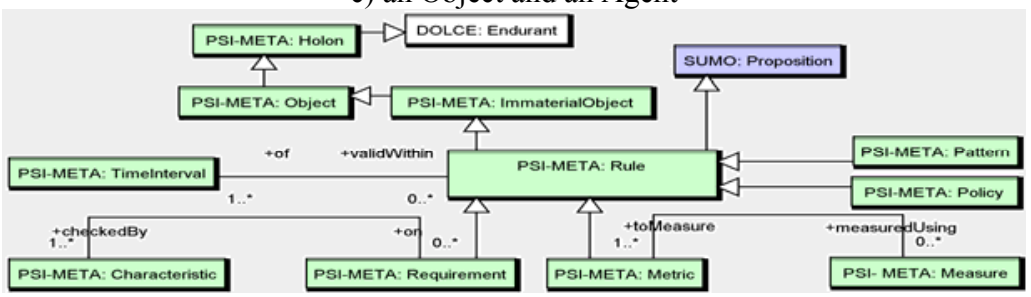

d) a Rule

Fig. 2. Semantic contexts of the key concepts.

may be suggested or prescribed by an industrial standard or a company policy. These prescriptions in terms of PSI Meta-Ontology are ProcessPatterns.

Any Process, as a pro-active stateful manifestation of a change in the Environment, is guided by its managing Agent to reach the State (Fig. 2b) of affairs in which the constituents of the Environment possess the properties partially or fully matching the Goal of that Agent. It is considered that a Process has reached its target State if such a state of affairs is reached. Otherwise the Process fails to reach its target State. A Goal, 
if complex, can be decomposed to simpler partial Goals as often done in problem solving. Such partial Goals are in fact the states of affairs that should be reached before the overall compound Goal can be attacked. States in PSI Meta-Ontology are the configurations of the constituents of an Environment. It is considered that a State is reached when the constituents of the Environment have properties with Values in the ranges satisfactory matching the corresponding Goal or partial-Goal of an Agent. In engineering design mentioned Goals are technologically controlled. For example, a technology of digital front-end design in microelectronics and integrated circuits prescribes that an overall Goal of a digital back-end design is the development of a design artifact in GDSII layout representation. At the same time the technology suggests that netlist, floorplan, placement and routing representations should be developed before the overall Goal can be reached. In these settings the States can be seen as technological milestones on the path through the problem solution space leading to the overall Goal. The requirements to the ranges of the property values of the constituents of the Environment are denoted by StatePatterns. StatePatterns are controlled by the Policies of a company which should be based on the standards of the particular industrial sector. Goals and corresponding partial Goals may be pursued by taking different alternative paths going through different States. If a problem solution space is represented as a directed graph, a State may have several alternative outgoing edges. These edges correspond to different admissible AtomicActions applying different changes to the Environment. A Decision on the choice of an admissible AtomicAction should be taken for choosing the continuation of the path at any State. In particular, a Decision in the target State chooses among the alternative to terminate the process in success and the alternative to refine the values of the properties of the constituents of the Environment heading to the same target State. Hence, a Decision is a specific AtomicAction which applies changes to an Environment indirectly - by choosing the alternative on the solution path. A Decision is also a mechanism to alter the course of the Process when the Goal or the sub-Goals are dynamically changed. In difference to an Environment, which is a Perdurant, a State is an Endurant because all its parts should be present at any TimeInstant of the presence of a State. A State is not a Holon because a State can not be a part of another State.

An Object (Fig. 2c) is a Holon which has Environment, belongs to an Environment, and may be changed in the course of the execution of an AtomicAction. An Object may have Characteristics, though the relationship of an Object to a Characteristic is not explicitly specified at this level of abstraction. The reason is that we refrain from letting the subclasses of an Object inheriting this very generic relationship. Instead we prefer to specify individual relationships between the subclasses of an Object and a Characteristic at lower abstraction levels - for example in the Core or the Extensions of PSI Suite. An Object could be either material or immaterial. MaterialObjects are those Objects which are physically or legally substantial in the sense that they possess tangible physical non-temporal properties like mass, color, shape, size, speed, usage right and can not be copied or duplicated without borrowing a definite amount of physical or legal ${ }^{5}$ substance for it. The law of

\footnotetext{
${ }^{5}$ By an odd term of "legal substance" we mean a legal permission to have an extra copy of an Object which is not a physical object in the sense of SUMO or DOLCE. A good example of such an Object is a software program with a license (legal substance).
} 
conservation of matter is applicable to material objects. In PSI Meta-Ontology MaterialObject subclasses are an Agent, a MaterialArtifact, a ConsumableResource, a Tool. ImmaterialObjects in contract to material ones are not substantial in physical or legal sense. Hence, they can be copied or duplicated without consuming physical or legal substance for that. In PSI Meta-Ontology ImmaterialObject subclasses are an ImmaterialArtifact, a Rule, a Plan, a Fact, a Belief.

An Agent (Fig. 2c) is a MaterialObject who possesses pro-activity, is able to execute AtomicActions and manage Processes. Pro-activity of an Agent is revealed in pursuing Goals of changing the Environment to a desired State. An Agent is the only entity which can change its Environment by executing AtomicActions applied to the Objects in the Environment. An Agent has Beliefs about its Environment(s) which are the hypotheses believed to be true. These Beliefs may further become Facts if confirmed by the happenings [4] perceived by the Agents. Beliefs together with desires and intentions are important basic elements forming the behavior of an Agent. This behavior is regulated by BehaviorPatterns specified as Rules. An Agent is an abstract entity which is a generic model for an individual person (a manager, a designer), a group of persons or artificial agents acting on behalf of physical persons (a team or an organizational unit), or an external pro-active entity influencing the Environment of an observed Process in a definite way. These aspects of an Agent are specialized and refined at the lower abstraction levels by PSI Core ontologies.

A Rule (Fig. 2d) is an ImmaterialObject which is a principle, a condition, a procedure, a generic pattern, or a norm regulating possible process, action, behavior, or state of affairs. As far as a Rule subsumes to a Holon it inherits structural parthood relationship of a Holon. Hence, a Rule may be an atomic proposition or a more complex composition of other Rules. As far as a Rule is an Endurant no temporal parthood relationships are allowed for its proper parts - the composition of a rule can not be changed in time. A Rule itself still has a temporal property of validity - it is valid within a particular TimeInterval or several particular TimeIntervals. If a Rule is a principle or condition that customarily governs behavior then it subsumes to WordNet: Rule and further on to SUMO: Proposition. If a Rule is a generalization that describes recurring facts or events then it subsumes to WordNet: Law and further on to SUMO: Proposition. If a Rule is something regarded as a norm constraining possible action or behavior then it subsumes to WordNet: Regulation and further on to SUMO: Proposition.

\section{Ontology Engineering Methodology}

The methodology used in the design of PSI Meta-Ontology may be identified as "shaker modeling". It is the combination of bottom-up and top-down modeling techniques exercised in subsequent design iterations. The source for the top-down activity is PSI Theoretical Framework. The source for the bottom-up phase is domain knowledge acquired from subject experts and formalized in the Core of PSI Suite of Ontologies. Both kinds of sources are refined in iterations before performing the phases of Meta-Ontology design. The sources are also aligned to the foundational reference ontology and mapped to the common sense reference ontology using PSI 
Meta-Ontology as upper-level semantic "glue" in the last two phases of every design iteration.

The most recent revision of the Theoretical Framework ${ }^{6}$ is used in the first phase skeleton design of the Meta-Ontology. The outcome is represented as a UML class diagram. Skeleton design phase is a top-down activity because a more abstract theoretical framework is used as a source for the development of a more elaborated descriptive theory. In the refinement phase the harmonization of the skeleton of the Meta-Ontology with the previous stable revision of the Core Ontologies of PSI Suite is performed. The objectives of this harmonization activity are: (i) ensuring that the upper-level model does not contain components which contradict to the core-level model in their semantics and (ii) ensuring that all core-level concepts are properly mapped to the upper-level concepts. Previous stable revision of PSI Core Ontolgies is used to ensure upward compatibility of the revisions of the PSI Suite comprising Meta-Ontology. The outcome of this phase is presented in the form of three separate UML class diagrams: (i) the taxonomical structure of the Meta-Ontology; (ii) the diagram of the "horizontal" relationships among the concepts of the Meta-Ontology; (iii) the mappings of the concepts of the Core Ontologies to the concepts of the MetaOntology. Refinement phase is a bottom-up activity because an upper-level model is harmonized with the lower-level one - the core part of the domain theory. At the beginning of the alignment phase Meta-Ontology is checked for the conformance to the ontological choices of the reference foundational ontology and its taxonomical structure is formally evaluated. As DOLCE is chosen as a reference foundational ontology for PSI, the upper-level model is checked for being descriptive, possibilistic, multiplicative, and perdurantistic. OntoClean [19] is used as a methodology for formal evaluation of the taxonomy. As result, the taxonomy is refined and formally evaluated. Further on, Meta-Ontology concepts are mapped to the reference ontology which has been chosen as a source of common sense semantics - SUMO+WordNet in our case. These mappings allow checking if our upper-level theory is sound enough to adequately conform to human beliefs about what the world is. If the result of such verification is positive (all the mappings are easily built and their semantics is easily understood), then we may expect that PSI Meta-Ontology will be accepted by humans without major difficulties. These common sense mappings may also be used as "referees" at the subsequent bridging phase. Bridging phase is actually not the phase of Meta-Ontology design. It is the activity in which Meta-Ontology is used as a semantic bridge to help evaluating the Core of PSI Suite against the other ontologies describing similar domain theories. For PSI Core such ontologies are the Enterprise ontology, TOVE ontology, and PSL ontology. PSI Core ontologies are mapped to them through PSI Meta-Ontology.

The iterations of PSI Meta-Ontology development are organized as shown in Fig. 3. The whole process is performed in two stages: (i) initial design and (ii) iterative refinement similarly to what is suggested by DILIGENT [20] methodology of collaborative ontology engineering. It may be stated that DILIGENT in our approach is used as the upper-level methodological framework organizing iterations in a needed way. DILIGENT is used because the development of our Suite of

${ }^{6}$ PSI Theoretical Framework v.2.0 [16] has been used in the design of PSI Meta-Ontology v.2.2 [18]. 
Ontologies is done in a distributed dynamic environment (several local groups of subject experts from different organizations in frame of PSI and PRODUKTIV+ projects take part).

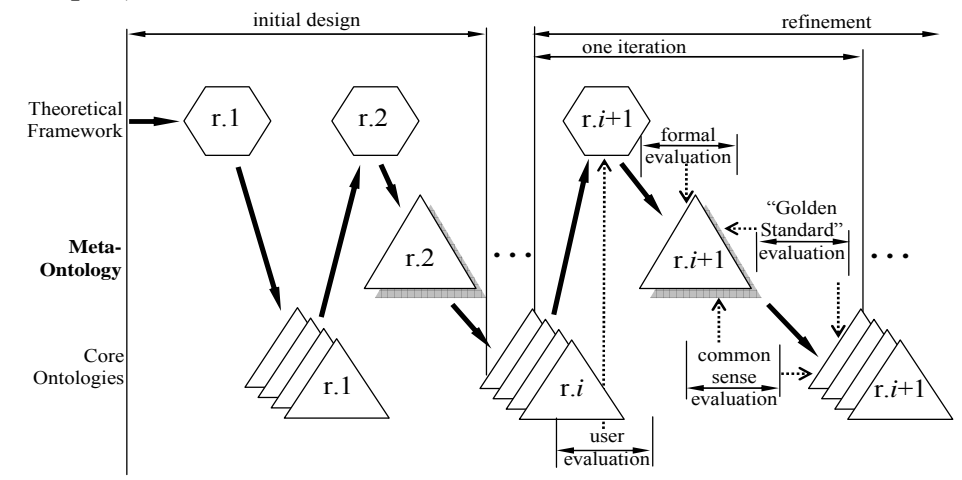

Fig. 3. Iterations of PSI Meta-Ontology design.

The stage of the initial design is the preparatory activity at the very beginning of ontology design process. Its objective is to develop the initial revisions of the Theoretical Framework and the Core set of the domain ontologies. An initial revision of the Meta-Ontology is developed at the end of the initial design stage because it requires both as sources. It is worth noticing that two revisions of the Theoretical Framework are developed before starting the design of the Meta-Ontology. The second revision is the result of the refinement based on the user evaluation feedback on the first revision of the Core ontologies. Hence, even the first revision of the MetaOntology is designed with the account for the user evaluation of the domain theory. An iteration of the refinement stage also uses the latest revision of the Theoretical Framework developed in this iteration and the revision of the Core ontologies built in the previous iteration. Iteration starts with the development of the Core set of ontologies based on the Meta-Ontology revision of the previous iteration and ends by the development of the new revision of the Meta-Ontology.

Several kinds of ontology evaluation activities are undertaken in each design iteration (Fig. 3). The first one is user evaluation. The objective of the user evaluation is to find out if the Core set of ontologies fits the requirements of user teams and the requirements of the software development based on this set of ontologies. An external evaluation by independent experts may also be done at this stage to ensure that evaluation results are unbiased and of good quality. It has been found out [21] that for PSI Core ontologies probably the best fitting methodology is Pinto and Martens [22]. The feedback of this iteration is taken into account in the refinement of the Theoretical Framework. The second kind of evaluation activity - formal evaluation, is undertaken at the Alignment phase after the newly developed revision of the MetaOntology has reached release candidate state. The objective of formal evaluation is to check the conformance of the taxonomy structure of the Meta-Ontology to metaproperties of rigidity, identity, and dependence [19]. The methodology for this kind of evaluation is OntoClean [19]. The results of the formal evaluation are used for the refinement of the release candidate of the Meta-Ontology. The third kind of evaluation activity is the evaluation of the Core set of ontologies and the Meta- 
Ontology. Similarly to the formal evaluation it is performed at the Alignment phase. The conformance of the Core set to the common sense is now checked. The mappings of the Core ontologies to the reference common sense ontology (SUMO) are elaborated using the Meta-Ontology as the "glue", like for example in [23]. The result allows estimating how easily domain experts may (potentially) commit to the Suite of Ontologies. If the Core set maps well to the common sense reference ontology one may expect that the commitment of domain experts to it may be reached considerably easily. If the mapping is bad then the ontology is either a novel extension of the common sense conceptualization or, more probably, is badly designed. The feedback of this kind of evaluation is used in refining the Core ontologies and, later on, in refining the next revision of the Meta-Ontology. Finally, the fourth kind of evaluation is the comparison of the Core set of ontologies with the so called "Golden Standard" [24]. By "Golden Standard" we mean a highly reputable ontology describing the theory of the same or a similar domain which has already gained broad commitment by domain experts. The evidence of such a commitment may be that a "Golden Standard" ontology is the basics for a standard, a de-facto standard, or a standardization proposal. This kind of evaluation is performed at the Bridging phase. Similarly to the common sense evaluation the mappings of the Core set of ontologies to a "Golden Standard" are built. However, the objective of the evaluation is different. Completeness and expressiveness of the Core Ontologies are checked at this time. If all the concepts of a "Golden Standard" ontology are mapped by the concepts of the Core set then it may be estimated that the Core set covers the domain equally to or better than a "Golden Standard". Otherwise, the core set is less complete than the "Golden Standard". In the latter case the reasons of potential incompleteness should be analyzed. In a safe case it may be found out that the domain described by the "Golden Standard", though similar to ours, is broader. Otherwise, the Core set is incomplete. The mappings in the opposite direction - from the concepts of a "Golden Standard" to the concepts of evaluated Core ontologies, may help assessing the level of the expressiveness of the target. For example, if all the concepts of the "Golden Standard" map to single concepts of the evaluated Core set then it may be the case that the Core set possesses at least the same level of expressiveness at the "Golden Standard".

\section{Ontology Implementation and Evaluation}

PSI Meta-Ontology v.2.2 [18] has been implemented in OWL-DL7 . PSI Theoretical Framework v.2.0 [16] and Core ontologies of PSI Suite of Ontologies v.2.1 [6] have been used as the knowledge sources. PSI Suite of Ontologies v.2.2 [7] has been developed based on the elaborated Meta-Ontology v.2.2. Two different kinds of evaluation have been accomplished for PSI Meta-Ontology so far: formal evaluation and commonsense evaluation. Besides that, user evaluation of the set of the Core ontologies v.2.1 has been done before the beginning of the development of the MetaOntology v.2.2, as described in Section 5. "Golden Standard" evaluation is still in

7 Web Ontology Language, http://www.w3.org/TR/owl-guide/. OWL-DL implementation of PSI Meta-Ontology is available at http://ermolayev.com/psi-public/psi-meta-v-2-2-draft.owl 
progress. User evaluation of the Core ontologies v.2.1 has been performed by the group of PSI software developers who used a goal-based evaluation routine to assess the appropriateness, the completeness, and the upward compatibility of the Suite of Ontologies. Appropriateness has been evaluated by checking if the Suite fulfils the requirements imposed by developed software. Completeness and upward compatibility with the previous revision has been checked by transferring the instances of the PSI knowledgebase v.2.0 to v.2.1. User evaluation revealed minor problems which have been immediately resolved allowing us to fix v.2.1. Several issues have been listed as the ones for the future development [6]. These issues have been taken into account in the revision of the Theoretical Framework v.2.0.

Formal evaluation of the taxonomy of PSI Meta-Ontology has been performed using OntoClean methodology [19]. The goal of taxonomy analysis is to verify if the structure of the taxonomy is formally correct. Other outcomes of this formal analysis are: (i) classifying taxonomy nodes according to OntoClean ontology of property types [25]; (ii) extracting the part of the analyzed taxonomy which is its backbone taxonomy [25]. In the course of this evaluation OntoClean meta-properties have been assigned to the concepts of the Meta-Ontology. After that OntoClean constraints have been applied to analyze if there are constraint violations in the taxonomy ${ }^{8}$. Applying OntoClean constraints to PSI Meta-Ontology subsumptions revealed no violations. Hence, the structure of the taxonomy of PSI Meta-Ontology is formally correct. Following [25], the concepts of PSI Meta-Ontology were classified according to OntoClean ontology of property types. As it has been found out, all PSI MetaOntology own concepts are Sortals. Non-sortal concepts are imported from DOLCE and are the categories forming the most upper part of the taxonomy. Among the sortals 16 are types and 17 are quasi-types. Categories, types and quasi-types form the backbone taxonomy of PSI Meta-Ontology. Among the remaining 6 concepts 5 are material roles and only 1 is a mixin. PSI Meta-Ontology does not contain phased sortals, formal roles and attributions. Backbone taxonomy of PSI Meta-Ontology and the parts of the ontology extending the backbone taxonomy are pictured in Fig. 1 using different shades of gray.

The objective of commonsense evaluation was to find out if the Meta-Ontology facilitates in mapping the Core ontologies to the reference foundational ontology. The mappings of the concepts of six PSI Core ontologies to WordNet+SUMO through PSI Meta-Ontology have been done using subsumptions [18]. It has been found out that using Meta-Ontology as semantic "glue" made these mappings more precise and facilitated to defining the semantics of the concepts of the Core more explicitly. For example, looking up for a Project (the concept of PSI Project ontology) in WordNet+SUMO ${ }^{9}$ reveals that a project is both: (i) "any piece of work that is undertaken or attempted" which subsumes to SUMO: IntentionalProcess and further to SUMO: Process; and (ii) "a planned undertaking" which subsumes to SUMO: Plan. The semantics of PROJECT: Project as specified in [7] reveals that a Project subsumes to PSI-META: Plan and consequently to SUMO: Plan. The mapping to SUMO: Process is therefore discarded as irrelevant. From the other hand, the analysis of the "hanging" concepts in the Meta-Ontology helps revealing the contexts in the

\footnotetext{
${ }^{8}$ Detailed description of the results of this formal evaluation is given in [18].

${ }^{9}$ KSMSA Ontology Browser has been used: http://virtual.cvut.cz/ksmsaWeb/browser/title
} 
Core which are still under-developed. For example, PSI-META: Goal does not subsume the concepts of the Core v.2.2. Therefore we may suspect that the aspects of goal-directed behavior in the Actor and Process Core ontologies v.2.2 still require refinement.

\section{Concluding Remarks and Outlook}

PSI Meta-Ontology is the upper level descriptive theory for the set of the Core ontologies of PSI Suite of Ontologies. PSI Suite is an interlinked modular library of ontologies describing the domain of engineering design performance in microelectronics. PSI Meta-Ontology is more domain-independent. It formalizes a meta-theory of stateful creative dynamic processes, pro-active agents, and objects situated in nested dynamic environments based on the formal representation of time, events, and happenings. This upper-level theory may be used as a meta-level for domain ontologies in different application domains having common features. PSI Meta-Ontology is designed as a semantic bridge formalizing the mappings of PSI Domain ontologies to abstract ontological foundations and common sense. It is also used as semantic "glue" for bridging PSI domain theory with other theories widely accepted in the domains where processes, states, and participating objects are the major entities. These mappings and semantic bridges are supposed to ease the commitment of potential users to PSI Suite. PSI Meta-Ontology is also used as a "proxy" for different kinds of evaluation of PSI ontologies in frame of our "shaker modeling" methodology for ontology refinement. In its current revision PSI MetaOntology is still lightweight in the sense that it does not provide rich axiomatic definitions of domain semantics. Enhancing it with formal axioms is the activity planned for future work. One more direction of our future development is extending the sphere of influence of PSI Meta-Ontology to cover the Extensions of our Suite of Ontologies. We are also interested in applying our upper-level ontological framework in the domains outside the scope of PSI and PRODUKTIV+.

\section{Bibliography}

1. Ermolayev, V., et al: PRODUKTIV+ Performance Ontology v.1.0. Reference Specification. Technical Report PSI-P-ONTO-TR-2007-3, 30.09.2007, VCAD EMEA Cadence Design Systems, GmbH, 63 p.

2. Sohnius, R., Jentzsch, E., and Matzke, W.-E.: Holonic Simulation of a Design System for Performance Analysis. In: Marik, V., Colombo, A.W., and Vyatkin, V. (Eds.) Proc. 3d Int. Conf. HoloMAS 2007, Sept. 3-5, 2007, Regensburg, Germany, 447-454 (2007)

3. Ermolayev, V.et al: Fuzzy Time Intervals for Simulating Actions. In: Kop, C. and Kaschek, R. (Eds.) Proc. UNISCON 2008, Apr. 22 - 25, 2008, Klagenfurt, Austria, 429-444 (2008)

4. Ermolayev, V. and Matzke, W.-E.: A Lightweight Ontology of Environments, Events, and Happenings for Semantic Web Agents. Submitted to ESAS@COMPSAC 2008, available at: http://ermolayev.com/eva personal/PS/PSI-E2H-ESAS-2008-draft.pdf

5. Ermolayev, V. et al: An Agent-Oriented Model of a Dynamic Engineering Design Process. In: Kolp, M. et al. (Eds.): Agent-Oriented Information Systems III. 7th International Bi- 
Conference Workshop, AOIS 2005, Utrecht, Netherlands, July 26, 2005, and Klagenfurt, Austria, October 27, 2005. Revised Selected Papers, 2006, 168-183

6. Ermolayev, V. et al: Performance Simulation Initiative. The Suite of Ontologies v.2.1. Reference Specification. Technical Report PSI-ONTO-TR-2007-1, VCAD EMEA Cadence Design Systems, GmbH (2007)

7. Ermolayev, V. et al: Performance Simulation Initiative. The Suite of Ontologies v.2.2. Reference Specification. Technical Report PSI-ONTO-TR-2007-5, 28.12.2007, VCAD EMEA Cadence Design Systems, GmbH, 131 p.

8. Niles, I. and Pease, A.: Towards a Standard Upper Ontology. In: Int. Conf. on Formal Ontologies in Information Systems (FOIS'01), vol. 2001, 2-9. ACM New York, NY, USA (2001)

9. Masolo, C. et al: WonderWeb Deliverable D18. Ontology Library (final). (2003)

10. Fellbaum, C. (ed.): WordNet: An Electronic Lexical Database, MIT Press, 1999.

11. Uschold, M. et al: The Enterprise Ontology. Knowledge Engineering Review, 13(1), 1998

12. Grueninger, M., Atefy, K., and Fox, M.: Ontologies to Support Process Integration in Enterprise Engineering. Computational \& Mathematical Organization Theory 6, 381-394, 2000.

13. Bock, C. and Gruninger, M.: PSL: A semantic domain for flow models. Software Systems Modeling (2005) 4: 209-231

14. Mika, P. et al: Foundations for Service Ontologies: Aligning OWL-S to DOLCE. WWW2004, May 17-22, 2004, New York, NY USA. ACM 1-58113-844-X/04/0005, 563572

15. Oberle, D. et al: DOLCE ergo SUMO: On Foundational and Domain Models in SWIntO (SmartWeb Integrated Ontology). Technical Report, AIFB, University of Karlsruhe, Germany (2006)

16. Ermolayev, V. et al: Performance Simulation Initiative. Theoretical Framework v.2.0. Technical Report PSI-TF-TR-2007-1, VCAD EMEA Cadence Design Systems, GmbH (2007)

17. Ermolayev, V. et al: A Strategy for Automated Meaning Negotiation in Distributed Information Retrieval. In: Y. Gil et al. (Eds.): ISWC 2005 Proc. 4th Int. Semantic Web Conf. (ISWC'05), 6-10 Nov., Galway, Ireland, LNCS 3729, pp. 201 - 215, 2005

18. Ermolayev, V. et al: Performance Simulation Initiative. Meta-Ontology v.2.2. Reference Specification. Technical Report PSI-ONTO-TR-2007-4, 12.01.2008, VCAD EMEA Cadence Design Systems, GmbH, 75 p, available at http://ermolayev.com/psi-public/PSIMETA-v-2-2-Spec.pdf

19. Guarino, N. and Welty, C.: Supporting ontological analysis of taxonomic relationships. Data and Knowledge Engineering, Vol. 39, No. 1, pp. 51-74, 2001

20. Vrandečić, D. et al: The DILIGENT knowledge processes. J. of Knowledge Management, 9(5), 2005, 85-96

21. Simperl, E.: Evaluation of the PSI Ontology Library. Technical Report, DERI Innsbruck, Austria, June, 2007, 13 p.

22. Pinto, H. S. and Martins, J. P.: A Methodology for Ontology Integration. In: Proc. Int. Conf. on Knowledge Capture (KCAP 2001), ACM Press, (2001)

23. Keberle, N., Ermolayev, V., and Matzke, W.-E.: Evaluating PSI Ontologies by Mapping to the Common Sense. In: Mayr, H. C, Karagiannis, D. (Eds.): Information Systems Technology and its Applications Proc. 6th Int. Conf. ISTA 2007, May 23-25, Kharkiv, Ukraine, 2007, GI LNI Vol 107, 91-104

24. Brank, J., Grobelnik, M., and Mladenic, D.: A Survey of Ontology Evaluation Techniques. In Proc. SiKDD, October 17, 2005, Ljubljana, Slovenia (2005)

25. Guarino, N and Welty, C. A.: A Formal Ontology of Properties. In: Dieng, R. and Corby, O. (eds.) Proc. EKAW 2000, Juan-les-Pins, France, Oct. 2-6, 97-112 (2000) 